\title{
Wettability-independent bouncing on flat surfaces mediated by thin air films
}

\author{
Jolet de Ruiter, Rudy Lagraauw, Dirk van den Ende and Frieder Mugele ${ }^{\star}$
}

\begin{abstract}
The impingement of drops onto solid surfaces ${ }^{1,2}$ plays a crucial role in a variety of processes, including inkjet printing, fog harvesting, anti-icing, dropwise condensation and spray coating $^{3-6}$. Recent efforts in understanding and controlling drop impact behaviour focused on superhydrophobic surfaces with specific surface structures enabling drop bouncing with reduced contact time ${ }^{7,8}$. Here, we report a different universal bouncing mechanism that occurs on both wetting and non-wetting flat surfaces for both high and low surface tension liquids. Using high-speed multiple-wavelength interferometry ${ }^{9}$, we show that this bouncing mechanism is based on the continuous presence of an air film for moderate drop impact velocities. This submicrometre 'air cushion' slows down the incoming drop and reverses its momentum. Viscous forces in the air film play a key role in this process: they provide transient stability of the air cushion against squeeze-out, mediate momentum transfer, and contribute a substantial part of the energy dissipation during bouncing.
\end{abstract}

The role of ambient air in drop impact and other dynamic wetting phenomena has long been neglected. Only recently, observations such as the suppression of splashing in drop impact at reduced ambient pressure ${ }^{10}$, the generation of splashes by superhydrophobic spheres falling into a liquid bath ${ }^{11}$, and the entrainment of air by fast-moving contact lines ${ }^{12}$ highlighted the relevance of this rather viscous ambient medium. For drop impact, theoretical studies ${ }^{13-16}$ suggested that splashing might be related to the presence of a thin lubricating air layer between the impacting drop and the substrate. Subsequent experiments confirmed the transient formation of an air layer with (sub)micrometre thickness ${ }^{9,17-20}$. However, it turned out that the air film collapses on a microsecond timescale for typical impact speeds of splashing experiments (of the order of $\mathrm{m} \mathrm{s}^{-1}$ ). However, as we report in this Letter, the air film remains intact if the initial impact speed is reduced to less than $v \sim 0.5 \mathrm{~m} \mathrm{~s}^{-1}$. In this case, the drop rebounds without ever directly touching the surface.

We release liquid drops of water, glycerol, silicone oil and various other organic liquids (Supplementary Table 1) of millimetric size $(R=0.52 \ldots 1.03 \mathrm{~mm})$ from a height of several millimetres to a few centimetres to fall onto carefully cleaned and dust-free surfaces of variable wettability (Methods). On their first impact the drops have initial Weber numbers $\mathrm{We}=\rho R \nu^{2} / \sigma=0.64 \ldots 4.3$ ( $\rho$ : liquid density; $R$ : drop radius; $\sigma$ : surface tension). For all liquids studied, side-view images taken with a high-speed video camera (Fig. 1a) show that the drops bounce provided that the impact speed is not too high. For water, the maximum impact speed is $0.48 \mathrm{~m} \mathrm{~s}^{-1}$, corresponding to $\mathrm{We}_{\max } \approx 4$. Throughout the entire bouncing process, the apparent contact angle observed in side-view images remains close to $180^{\circ}$, both for clean glass substrates with an equilibrium contact angle $\theta_{\mathrm{Y}}=3^{\circ}$ (Fig. 1a) and for hydrophobized surfaces with $\theta_{\mathrm{Y}}=90^{\circ}$ (Supplementary Movie 2). This remarkable independence of liquid-substrate interaction strongly suggests that the liquid is not in direct contact with the solid. A more detailed view of the drop-substrate interface is obtained from bottom-view images recorded through the transparent substrate using monochromatic light (Fig. 1b and Supplementary Movies 9-11). Interference fringes observed throughout the entire bouncing process (Fig. 1c) reveal the continuous presence of an air layer between drop and substrate. Simultaneous recording of interference fringes at two wavelengths allows the conversion of the interference patterns to absolute air film thickness, Fig. 1d (see ref. 9 and Methods). For the maximum impact speed, the air film thickness decreases to a minimum value of $h_{\min } \approx 200 \mathrm{~nm}$ during bouncing.

After rebounding from the surface the drop flies vertically upwards until it is slowed down to rest by gravity at its maximum height, then falls back onto the substrate. Because of finite energy losses in the bouncing process, the maximum height, and hence the speed of the subsequent impact process, are slightly lower than during the first impact. (Based on the impact velocities $v$ and $v^{\prime}$ before and after the bounce, we find a restitution coefficient $\varepsilon=v^{\prime} / v \approx 0.95$, see Supplementary Fig. 3.) As a consequence, the drop bounces again. This leads to a cascade of consecutive bouncing events at progressively reduced impact speed (Fig. 2a). Effectively, each subsequent impact is a separate experiment, in which the drop is gently released from a slightly lower initial height, leading to a correspondingly lower impact speed. (Like in the case of the initial release from the needle, the drop has some finite vibrational energy ${ }^{21}$, typically no more than $5 \%$ of the potential energy (see below). Except for a minor variation of the restitution coefficient, these weak oscillations do not noticeably affect the bouncing behaviour.) Again, the occurrence of repeated bouncing and the gradual loss of energy are independent of the wettability of the substrate (Supplementary Fig. 1).

During each impact, a new air film is formed, on which the drop spreads, recedes, and rebounds again. Eventually, when We has dropped below $\sim 0.02$ in a bouncing series, the water drop no longer visibly detaches from the substrate in side-view images, but simply oscillates. However, the centre of mass still moves up and down and the air film thickness derived from the interference images periodically increases and decreases as well. If we keep using the centre of mass velocity of the drop during this 'hovering' phase (Fig. 2a) to define an impact velocity, air-film-mediated bouncing is found to persist down to $v \approx 0.02 \mathrm{~m} \mathrm{~s}^{-1}$, corresponding to $\mathrm{We} \approx 0.004$. As the minimum air film thickness gradually decreases during hovering, the air film eventually collapses and the drop wets the surface (see the inset photo in Fig. 2a). 

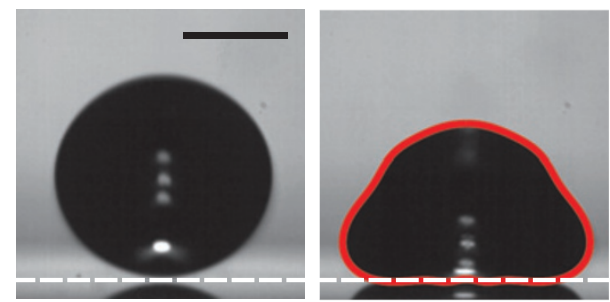

b

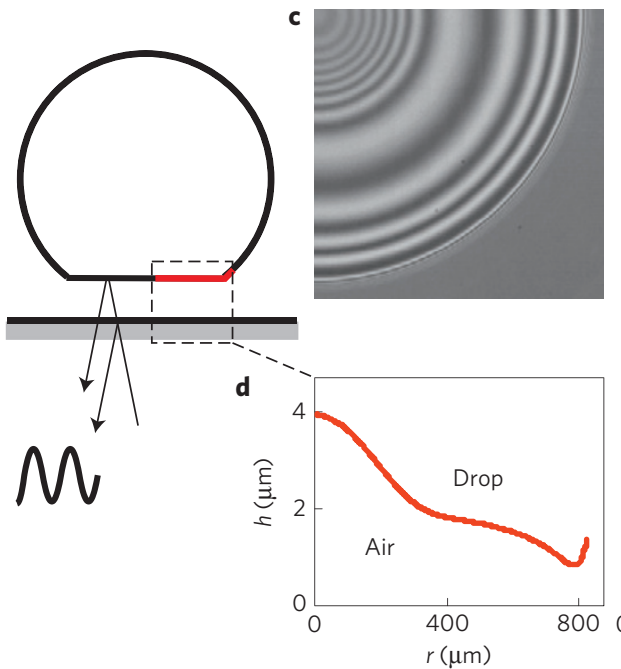

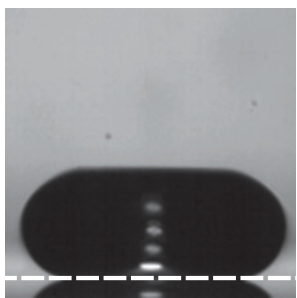
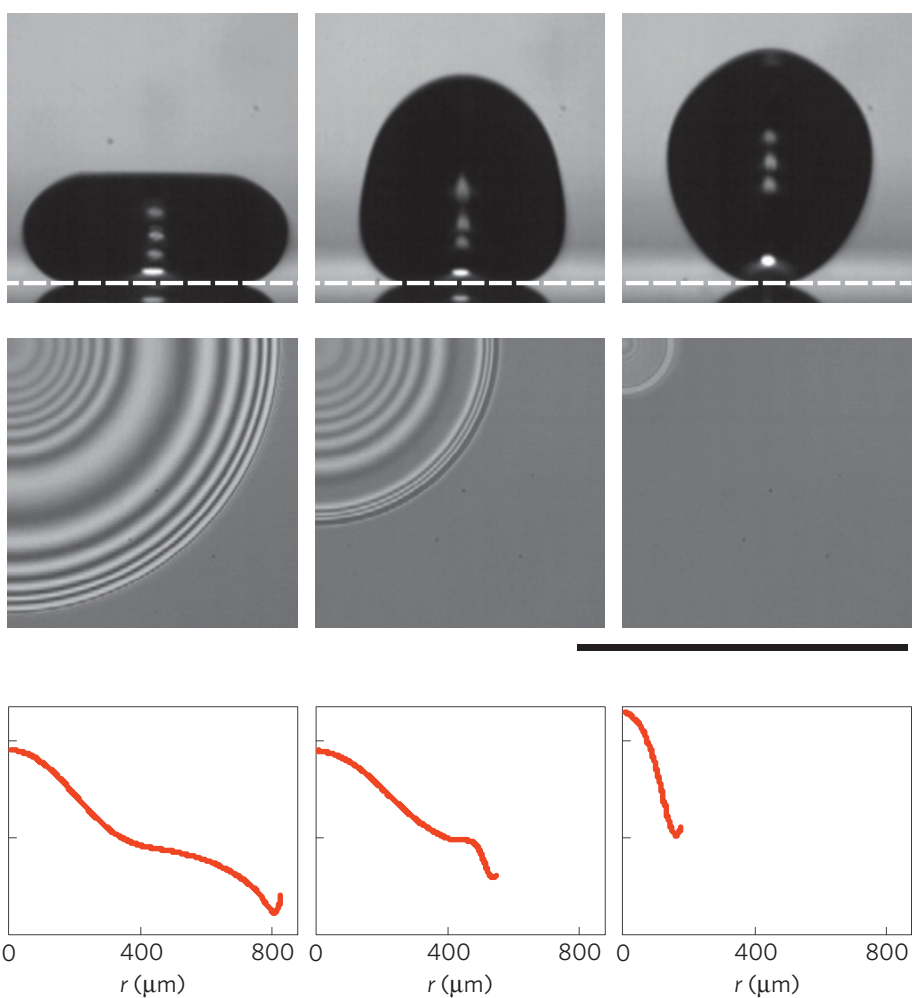

Figure 1 | Water drop bouncing off a glass substrate. a, Side-view images of a water drop impacting at $v=0.22 \mathrm{~m} \mathrm{~s} \mathrm{~s}^{-1}$. Young's angle: $\theta$ Y $=3^{\circ}$. Red line: shape mode decomposition $(n \leq 10)$. b. Sketch of the reflection interference microscopy method used to obtain the interference patterns shown in $\mathbf{c}$ (a quarter pattern is shown). The analysed section of the drop-air interface is shown in red. $\mathbf{d}$, Resulting radial profiles of the air film thickness. Note the different scales on the $x$ - and $y$-axes. Snapshots are taken left to right at $0 \mathrm{~ms}$ (reference; a only), 2.3, 4.0, 7.5 and 9.0 ms. Scale bars: 1 mm.

The same general phenomenology is observed for all surfaces and all liquids investigated, including, in particular, low surface tension decane, silicone oil and Fluorinert FC-40 (see Supplementary Table 1 and Movies 6-8). For all systems, air-film-mediated bouncing and hovering are found within a very similar range between $\mathrm{We}_{\min } \sim \mathrm{O}\left(10^{-3}\right)$ and $\mathrm{We}_{\max } \approx 2.2 \ldots 4.3$ (Fig. 3). The narrow range of $\mathrm{We}_{\max }$ suggests the existence of a characteristic minimum air film thickness $h_{\min }$ limiting the occurrence of airfilm-mediated bouncing. We can rewrite the theoretical prediction ${ }^{14}$ as $h_{\min }=5 R \mathrm{Oh}_{\mathrm{g}}^{8 / 9} \mathrm{We}^{-10 / 9}$, with $\mathrm{Oh}_{\mathrm{g}}=\mu_{\mathrm{g}}(\sigma \rho R)^{-1 / 2}$ being the Ohnesorge number, giving the ratio of gas viscosity $\left(\mu_{\mathrm{g}}\right)$ to surface tension and inertia. The prefactor $R \mathrm{Oh}_{\mathrm{g}}^{8 / 9}$ varies by less than a factor two for the various drops in our experiments (Supplementary Table 1). This leads to a prediction of $h_{\min } \approx 200-400 \mathrm{~nm}$ for maximum impact speed, largely independent of the liquid and consistent with the interferometry measurements.

Bouncing processes of drops involving thin air films were observed before in two special cases, namely vibrating liquid substrates $^{22-25}$ and gradually evaporating Leidenfrost drops on heated substrates ${ }^{21,26,27}$. In both cases, an external process-shaking or evaporation-above a certain threshold strength is required to sustain the air film and to enable bouncing. In contrast, in the present situation the film is stabilized by purely dissipative forces owing to viscous squeeze-out of the air underneath the drop. Compressibility effects can be neglected for the present impact speeds because the excess pressure in the air film is low, as discussed earlier using a balance between drop inertia and gas pressure ${ }^{14}$.

Bouncing generally requires the transient transformation of translational kinetic energy into elastic potential energy and back. Although the transient storage of energy in deformation is similar to conventional bouncing on superhydrophobic surfaces ${ }^{28}$, the challenge is to understand how the purely viscous squeeze-out forces in the air film can mediate the largely elastic rebound with a restitution coefficient $>0.9$. To do so, we analyse the flow and loss of energy and momentum throughout the process. We decompose the drop shapes extracted from side-view images of the entire bouncing series into Legendre polynomials $P_{n}$, the eigenfunctions of freely oscillating drops for $n=0 \ldots 10$. From this decomposition, we obtain the exact drop size, the position of the centre of mass $y_{\mathrm{CM}}$, and the coefficients $c_{n}$ of each eigenmode. As expected, the lowest modes dominate $(n \leq 4)$, with $c_{n}<0.1 R$ throughout the flight phase. This allows the extraction of the total centre of mass $E_{\mathrm{CM}}$ and internal vibrational $E_{\text {int }}$ energies-split into potential and kinetic contributions-and dissipation due to internal flow using well-known expressions for linearized drop oscillations (Methods). During bouncing, drop deformations are more pronounced than during flight, with transient values up to $c_{2} \approx 0.5 R$ for $\mathrm{We}>1$.

From $y_{\mathrm{CM}}$ we extract the acceleration $\ddot{y}_{\mathrm{CM}}$ and the net external force $F(t)=m\left(\ddot{y}_{\mathrm{CM}}(t)+g\right)$ acting on the drop ( $m$ : drop mass; $g$ : gravitational acceleration). Whereas $F$ vanishes as expected during the flight phase, we find a positive force throughout the entire bouncing phase, with a minimum around the moment of maximum drop deformation (Fig. 2b). This observation implies a subtle coupling between the viscous squeeze-out of the air film and the deformation of the drop that is crucial for the bouncing process: for a rigid object such as a sphere or disk inside a viscous medium the force required to move perpendicular to a solid wall is always oriented opposite to the direction of motion, implying a reversal of the force as soon as the object moves away from the surface. This suppresses bouncing. For bouncing drops, the situation is evidently different. The origin of the efficient positive momentum transfer can be traced to a distinct asymmetry of the 


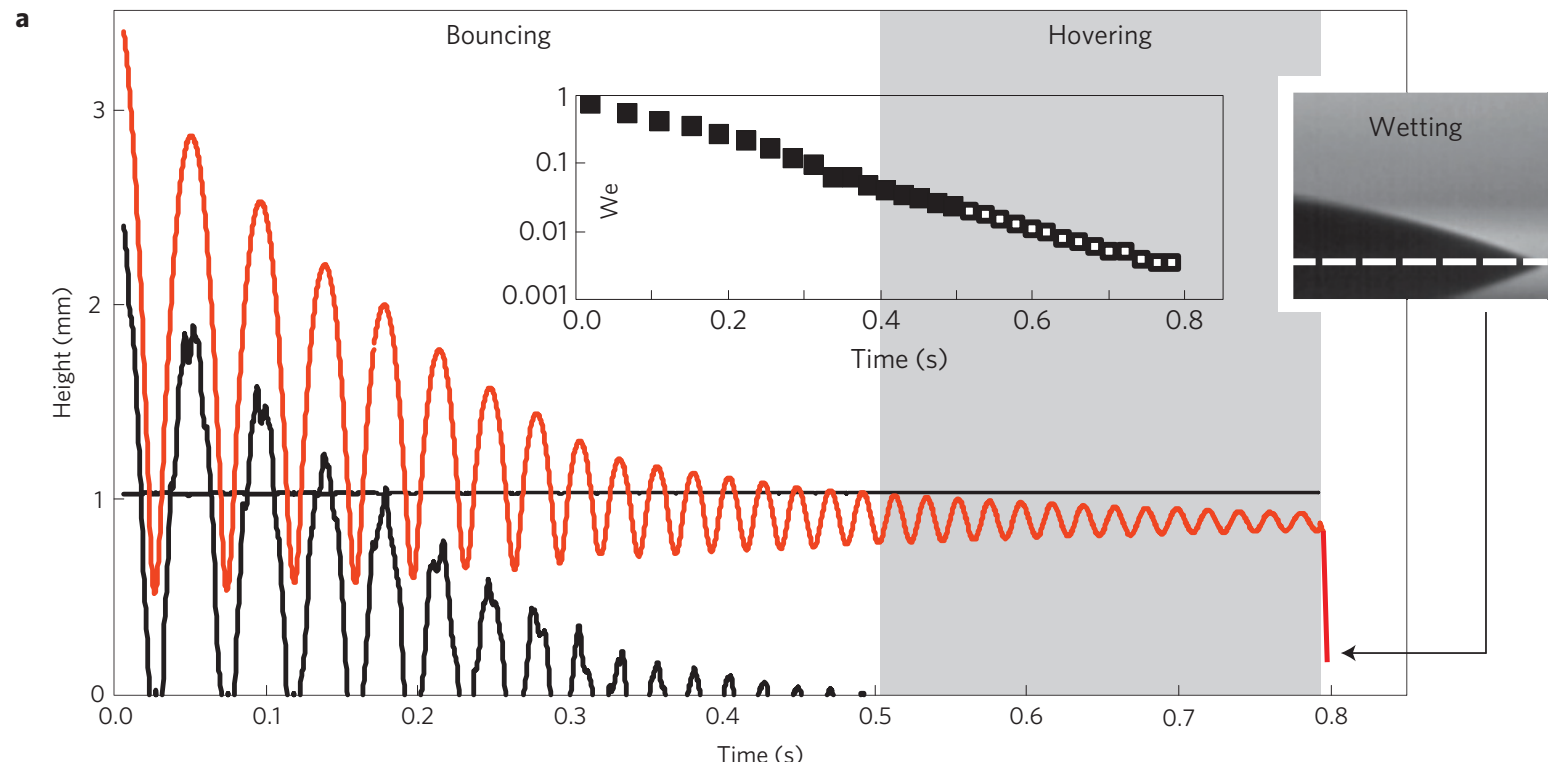

b

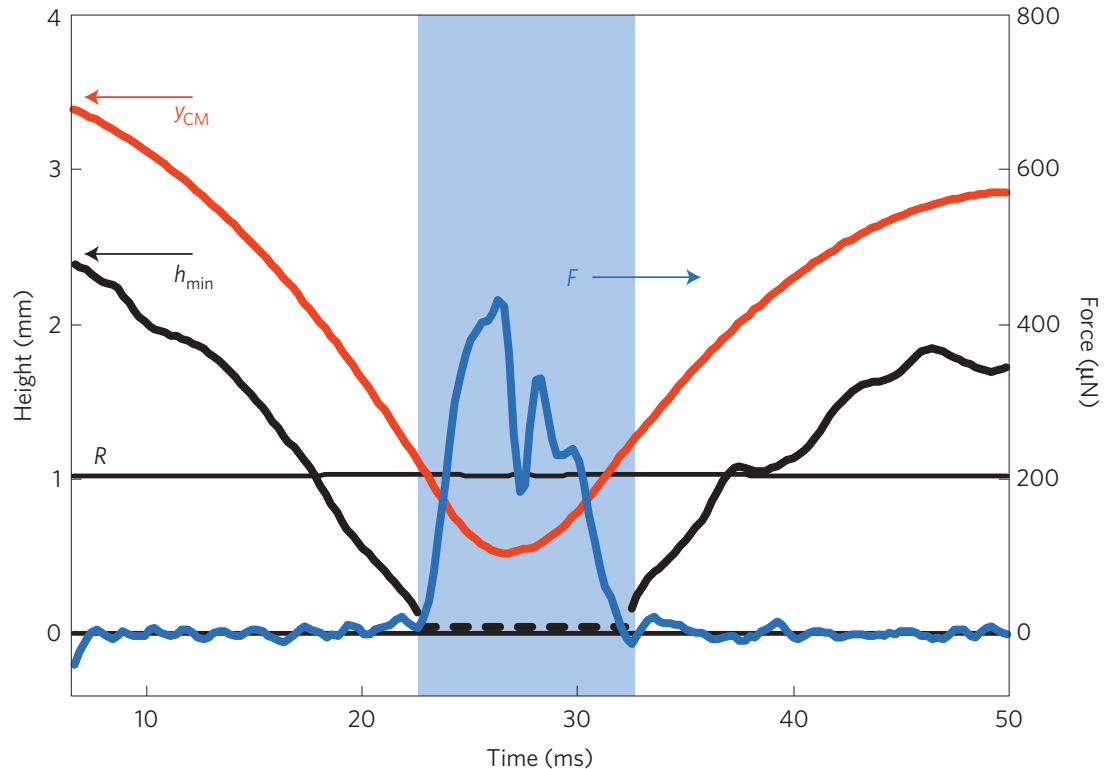

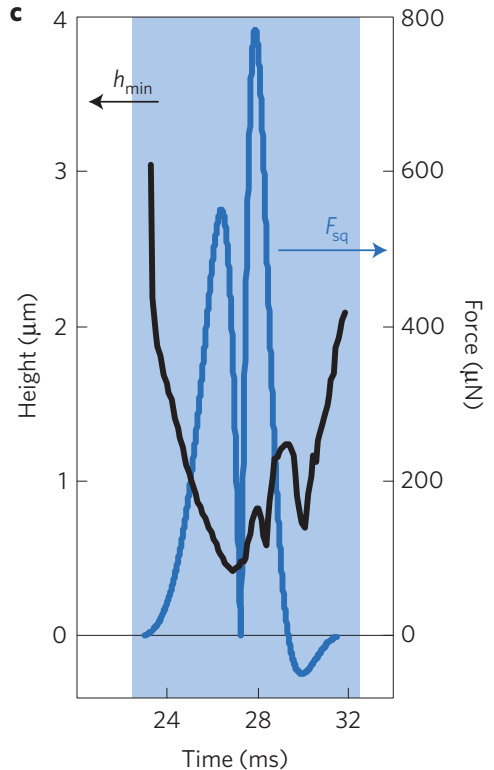

Figure 2 | Bouncing dynamics. a, Full bouncing series of an $R=1.03 \mathrm{~mm}$ water droplet on a smooth hydrophilic glass wafer that is fully wetted on

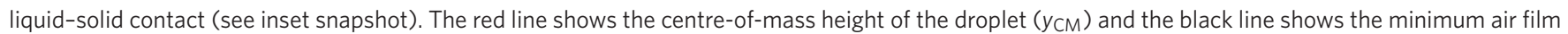
height $\left(h_{\min }\right)$. The inset shows the inherent decrease of We during the experiment. The grey shaded area indicates the final hovering phase. $\mathbf{b}, \mathbf{c}$, Details of the first bounce in $\mathbf{a}$. b. Centre-of-mass height of the droplet and the minimum air film height (as in a) and drop-substrate interaction force $F(t)=m\left(\ddot{y}_{\mathrm{CM}}(t)+g\right)$ (blue, right axis) based on side-view images. c, Minimum air film height $h_{\min }$ (black) obtained based on the interference microscopy

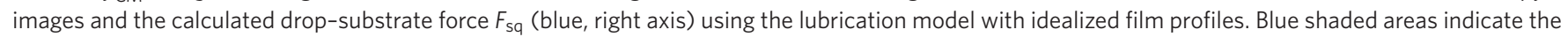
interaction phases.

air film profile between the spreading and the receding phase of the bouncing process (Fig. 4a). A sombrero-shaped dimple forms early on in the impact process and the drop gradually spreads, while the inner part of the dimple remains almost stationary (left panel). On retraction, the sharp kink at the edge of the drop moves radially inwards, while the total volume of the entrapped air film continues to decrease. The continuous net outward flux of air implies a positive average pressure throughout the entire bouncing phase. To quantify this effect, we numerically calculate the pressure profile in the film based on a lubrication flow for an idealized profile (Methods) that mimics the essence of the kink motion (Fig. $4 \mathrm{a}$ (inset) and b). The resulting integrated force is indeed positive throughout the entire bouncing process and exhibits a characteristic minimum around maximum drop extension (blue line in Fig. 2c) in almost quantitative agreement with the force obtained from the drop acceleration (blue line in Fig. 2b).

Energy dissipation in drop bouncing has been attributed to viscous damping of bouncing-induced oscillations during the flight phase $^{28}$. Dissipation during the bouncing phase is believed to be negligible, as contact line dissipation is suppressed, and bulk dissipation is negligible owing to the short 'contact' time. In contrast, we find that the primary energy loss in air-film-mediated bouncing occurs during the bouncing phase. After each rebound, most of the deformational energy is converted back into kinetic energy of the centre of mass $E_{\mathrm{CM}}$ (red line in Fig. 5). The small fraction of residual vibrational energy $E_{\text {int }}$ (blue line in Fig. 5) gradually decreases during 


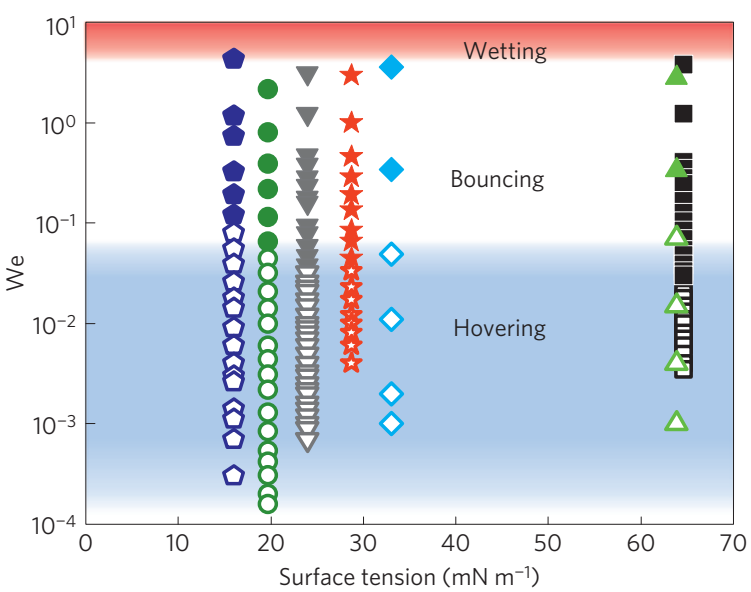

Figure 3 | Universality of air-film-mediated bouncing. Occurrence of bouncing (filled symbols) and hovering (open symbols) for various polar and nonpolar drops on glass: (black squares) water (with fluorescent dye); (green up triangles) $85 \mathrm{wt} \%$ glycerol; (cyan diamonds) sunflower oil; (red stars) $90 \mathrm{wt} \%$ propanol; (grey down triangles) decane; (olive circles) silicone oil; (blue pentagons) Fluorinert FC-40. Red shaded area: high-speed impacts leading to immediate wetting due to air film collapse; white area: bouncing; blue shaded area: hovering.

the flight phase, in close agreement with the viscous dissipation

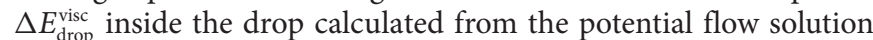
in the limit of low viscosity (Methods). Accumulated over an entire

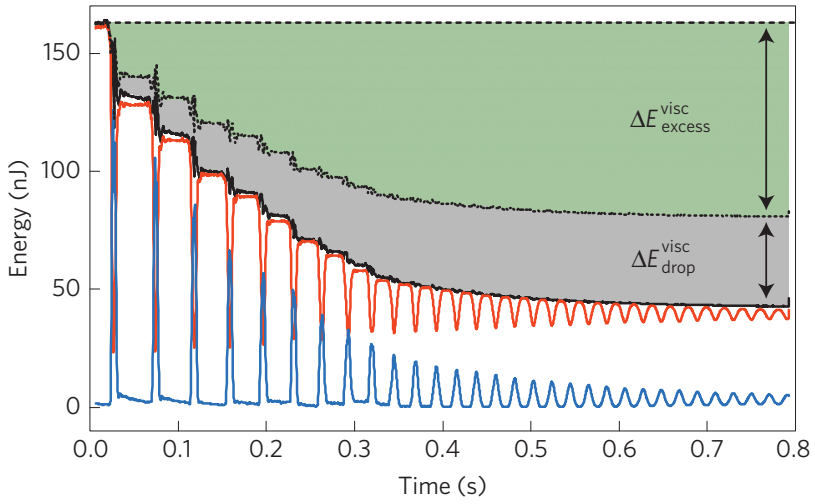

Figure 5 | Energy conversion and dissipation. Energy conversion for the bouncing series shown in Fig. 2: (red line) centre of mass energy $E_{\mathrm{CM}}$; (blue line) internal energy $E_{\text {int }}$; (black solid line) total energy $E_{\text {tot }}=E_{\mathrm{CM}}+E_{\text {int }}$ according to shape mode analysis; (black dotted line) $E_{\text {tot }}+\Delta E_{\text {drop; }}^{\text {visc }}$ (grey shaded) internal viscous dissipation; (green shaded) $\Delta E_{\text {excess }}^{\text {visc }}$ excess dissipation during bouncing due to air film dissipation and nonlinear viscous dissipation. Black dashed line: total initial energy in the system.

bouncing series, dissipation during flight amounts to no more than $\sim 20 \%$ of the total dissipation. After each bouncing event, however, the sum of the total energy $E_{\text {tot }}=E_{\mathrm{CM}}+E_{\text {int }}$ and $\Delta E_{\text {drop }}^{\text {visc }}$ exhibits a stepwise decrease (dotted black curve in Fig. 5; $\Delta E_{\text {drop }}^{\text {vis }}$ is shaded grey). The green shaded area thus corresponds to the unaccounted excess dissipation during bouncing, $\Delta E_{\text {excess }}^{\text {visc }}$. In part, this excess
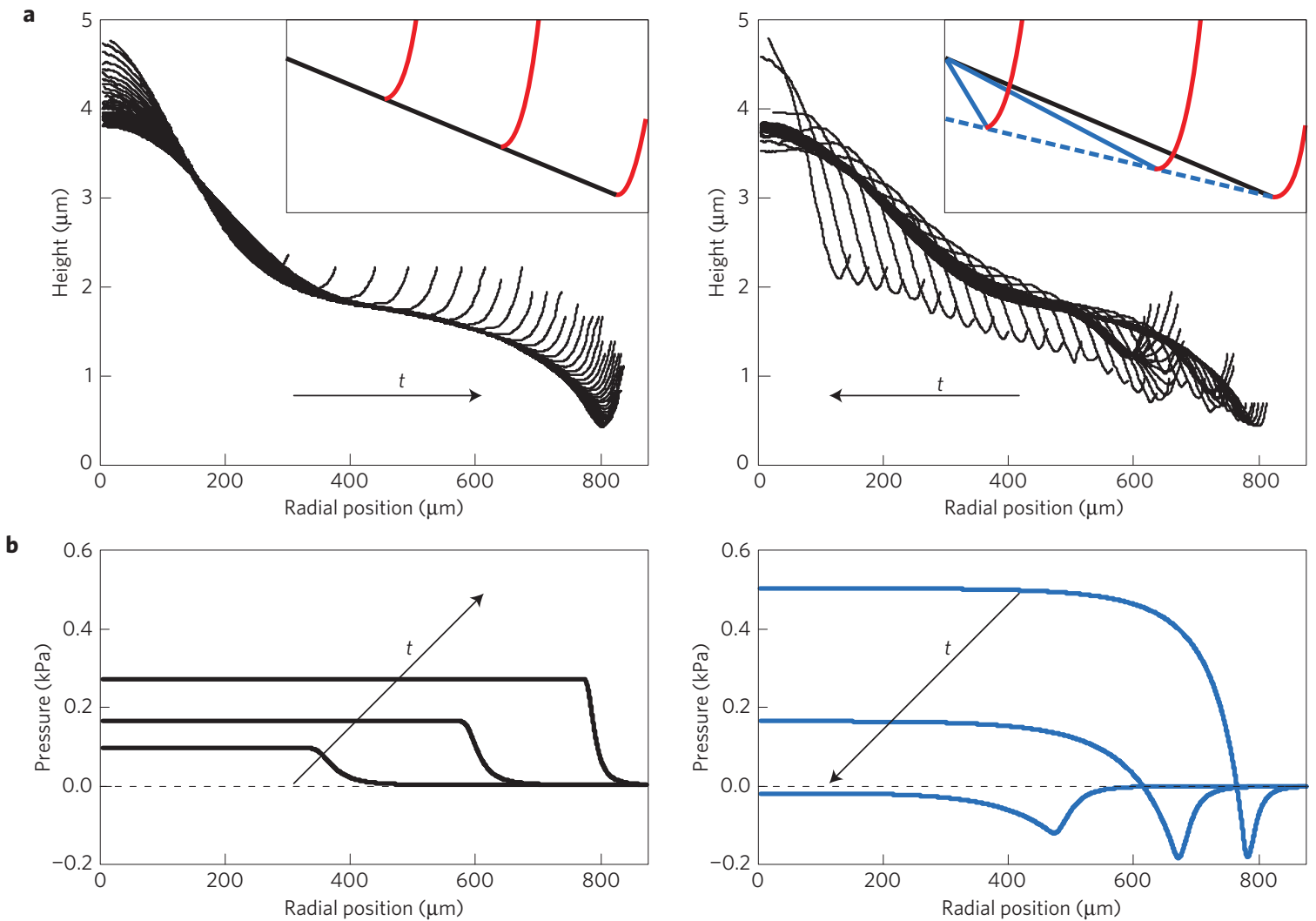

Figure 4 | Role of air film asymmetry. a, Time evolution of the air film thickness obtained by reflection interference microscopy for (left) the spreading phase up to $3.6 \mathrm{~ms}$ and (right) the contraction stage up to $8.6 \mathrm{~ms}$. Impact of an $R=1.03 \mathrm{~mm}$ water droplet with an initial velocity of $0.22 \mathrm{~m} \mathrm{~s}^{-1}$. Profiles are shown with $\Delta t=0.1 \mathrm{~ms}$. Inset: idealized air film profiles used for the numerical lubrication model: (red) parabolic outer kink profile, (black and blue) linear inner profile during spreading and contraction, respectively. b. Calculated pressure distributions for idealized air film profiles at representative time steps, as in $\mathbf{a}$. The time-order of the steps is indicated by the arrows. 
dissipation may be due to a failure of the linear approximation for larger deformations during the bouncing phase. However, the degree of deviation suggests the presence of an additional hitherto neglected dissipation channel: obviously, the viscous squeeze-flow in the air film that mediates the momentum reversal also involves viscous dissipation. A standard lubrication calculation (Methods) using the experimental film profiles allows the time-dependent local dissipation rate to be determined within the air film (Supplementary Fig. 4). Integrating these local rates over time and the drop-substrate interface shows that the total air film dissipation indeed amounts to a substantial fraction of the energy loss in Fig. 5. A conservative estimate yields that air film dissipation accounts for several per cent of the residual energy loss for the first high-We impacts and for almost the complete loss during later low-We bouncing events.

Air-film-mediated bouncing thus enhances the range of systems amenable to drop bouncing from high surface tension liquids on non-wetting surfaces to a much wider class of systems, including wetting surfaces and low surface tension liquids. Further experiments with plasma-treated polymer surfaces (Methods) indicate that air-film-mediated bouncing also persists for (super)hydrophobic surfaces exhibiting nano-roughness for roughness amplitudes below $100 \mathrm{~nm}$ (Supplementary Movie 12). This observation suggests that the benefits of air cushions can be exploited in combination with nano-texturing of liquid-repellent surfaces-for example, to minimize undesired solid-liquid contact even further.

\section{Methods}

Experimental set-up and procedures. Drops of water, $85 \mathrm{wt} \%$ glycerol (Sigma-Aldrich) in water, sunflower oil (commercial kitchen oil), $90 \mathrm{wt} \%$ propanol (Merck KGaA) in water, $n$-decane (Merck KGaA), silicone oil ( $\mu=5 \mathrm{mPas}$, Aldrich) and Fluorinert FC-40 (Sigma) were quasistatically dispensed from a syringe needle and detached by their own weight to obtain a uniform radius (see Supplementary Information for fluid properties). They reach an initial impact velocity of $0.22-0.49 \mathrm{~m} \mathrm{~s}^{-1}$. Trace amounts of fluorescent dye (rhodamine B isothiocyanate, Sigma) were added to the liquids if possible to suppress light reflections in the interferometry measurements ${ }^{9}$. Drops impact onto fully wettable polished glass wafers (RMS roughness $<3 \mathrm{~nm}$, as verified by atomic force microscopy) or glass wafers hydrophobized with a ultraviolet-cured silicone oil layer ${ }^{29}$ to obtain a water contact angle of $\sim 90^{\circ}$. The glass wafers were rigorously cleaned and plasma-treated to remove all irregularities-for example, dust particles-and transferred to a closed cuvette just before the impact experiment. In addition, superhydrophobic surfaces were produced by oxygen plasma etching of a $\sim 5 \mu$ m-thick photoresist layer (SU-8) on a glass wafer, which was subsequently coated with a $\mathrm{C}_{4} \mathrm{~F}_{8}$ layer to obtain an advancing contact angle of $155^{\circ}$ and a roughness-dependent receding contact angle of 120-150 (ref. 30). This procedure led to an RMS roughness varying between 8 and $100 \mathrm{~nm}$. All experiments were performed at room temperature and ambient pressure and recorded with three synchronized high-speed cameras. Full bouncing sequences were recorded from the side at 4,000 fps (Photron-FASTCAM Ultima 512). The air film was imaged through the transparent substrate by reflection interference microscopy using two wavelength distributions around 546 and $436 \mathrm{~nm}$, which were recorded separately at 20,000 fps with two high-speed cameras (Photron SA3 and SA5). The combination of dual wavelength and the broad bandwidth of the optical filters allows measurement of the absolute thickness of the air films with a spatial resolution better than $30 \mathrm{~nm}$ (ref. 9).

Image analysis. The time-dependent droplet contour $R(t, \theta)$ obtained from the side view using Laplacian edge detection is decomposed into Legendre polynomials $P_{n}(x)$, giving $R(t, \theta)=R_{0}+\sum_{n=0}^{\infty} c_{n}(t) P_{n}(x)$ with $x=\cos \theta$. Modes up to $n=10$ are included to obtain a sufficiently flat bottom when the droplet interacts with the substrate. (The lower modes dominate the energy dissipation, which converges quickly when including modes higher than $n=5$.) The evolution of the droplet shape is now fully characterized by the coefficients

$c_{0}(t), c_{1}(t) \ldots c_{10}(t)$, with $c_{n}(t)=((2 n+1) / 2) \int_{-1}^{1}\left\{R(t, \theta)-R_{0}\right\} P_{n}(x) \mathrm{d} x$ (see also ref. 31). The coefficient $c_{1}$ is chosen such that the centre $y_{0}(t)$ with respect to which $R(t, \theta)$ is determined is equal to $y_{\mathrm{CM}}(t)$.

Drop internal energy and dissipation. The internal vibrational energy can be found in terms of the drop shape decomposition ${ }^{32}$. The potential surface energy can be expressed up to second order in the shape coefficients $c_{n}$, as

$U_{\text {int }}=2 \pi \sigma \sum_{n=2}^{\infty}(((n-1)(n+2)) /(2 n+1)) c_{n}^{2}$. The kinetic energy and dissipation depend on the details of the internal flow field. Because viscosity plays a

negligible role and the flow is incompressible, we use potential theory, where the velocity potential is given by $\varphi(r, \theta)=\sum_{n=1}^{\infty} A_{n} r^{n} P_{n}(x) . A_{n}$ is the strength of mode $n$ and can for small interface deformations be expressed as its corresponding shape mode coefficient, $\dot{c}_{n}=n A_{n} R_{0}^{n-1}$. For large amplitudes of the fundamental mode $c_{2}>0.1 R$ (that is, during each bounce phase except the very last hover oscillations) energy is transferred between modes, leading to nonlinear coupling. Here, we will restrict ourselves to the linear approximation using the $c_{n}$ values during the bouncing phase as an estimate of the flow field. Then, the kinetic energy can be expressed as $K_{\text {int }}=2 \pi \rho R_{0}^{3} \sum_{n=1}^{\infty}(1 /(n(2 n+1))) \dot{c}_{n}^{2}$. In the limit of small viscosity the dissipation rate in the flow can be expressed as $\dot{E}_{\text {drop }}^{\text {visc }}=8 \pi \mu R_{0} \sum_{n=1}^{\infty}((n-1) / n) \dot{c}_{n}^{2}$. The latter two expressions are strictly valid only in flight, but are also used as an estimate during the interaction phases. The potential surface energy exhibits only a modest (10\%) difference between the linear approximation and the full expression.

Lubrication analysis. The flow in the squeezed air layer can be described in the lubrication approximation owing to its large $w / h \approx 1,000$ aspect ratio. In the incompressible regime, continuity leads to a radial volumetric flow rate $\dot{Q}(r)=-2 \pi \int_{0}^{r} \operatorname{sh}(s) \mathrm{d} s$ for the radial-dependent interface shape $h(r, t)$. Integration of the reduced Stokes equations, $\partial_{r} p=\mu \partial_{z}^{2} v$ and $\partial_{z} p=0$, yields a parabolic flow profile $v(r, z)=(3 \dot{Q}(r)) /(\pi r h(r))\left\{(z / h(r))-(z / h(r))^{2}\right\}$, assuming no-slip conditions. The dissipation rate $\dot{W}_{\mathrm{sq}}=\int \mu\left(\partial_{z} v\right)^{2} \mathrm{~d} V$ is given by $\dot{W}_{\mathrm{sq}}=(6 \mu / \pi) \int_{0}^{R_{\max }}\left(\dot{Q}^{2}(r)\right) /\left(r h^{3}(r)\right) \mathrm{d} r$, and the excess pressure by $\Delta p(r)=p(r)-p_{\infty}=(6 \mu / \pi) \int_{r}^{\infty}(\dot{Q}(s)) /\left(s h^{3}(s)\right) \mathrm{d} s$, where $p_{\infty}$ is the ambient pressure outside the squeezed layer. The squeeze force can be determined by integrating the pressure over the film area, leading to

$F_{\text {sq }}=6 \mu \int_{0}^{\infty}(s \dot{Q}(r)) /\left(h^{3}(r)\right) \mathrm{d} r$. The dissipation can be calculated from the experimental air film profile-at least, the fraction dissipated in the observable film region. To calculate pressure field and force, we use idealized film profiles with a linear profile on the inside matched to a parabolic profile outside the kink (with respect to the kink: $\Delta h=a(\Delta r)^{2}, a=1.45 \times 10^{-3} \mu \mathrm{m}^{-1}$ ). As shown in the insets of Fig. $4 \mathrm{a}$, the required asymmetry is introduced into the calculation by approaching the kink towards the surface from $[r, z]=[0,4]$ to $[800,0.4] \mu \mathrm{m}$ during the spreading phase, and by retracting it from there towards $[0,3.665] \mu \mathrm{m}$, both with a parabolic time dependence of the kink height. The dimple centre remains at a height of $4 \mu \mathrm{m}$. This geometry decelerates the drop during the spreading phase to a complete stop and reproduces the observed restitution coefficient.

Received 24 March 2014; accepted 3 October 2014; published online 10 November 2014

\section{References}

1. Yarin, A. Drop impact dynamics: Splashing, spreading, receding, bouncing.... Annu. Rev. Fluid Mech. 38, 159-192 (2006).

2. Marengo, M., Antonini, C., Roisman, I. V. \& Tropea, C. Drop collisions with simple and complex surfaces. Curr. Opin. Colloid Interface Sci. 16, 292-302 (2011).

3. Van Dam, D. \& Le Clerc, C. Experimental study of the impact of an ink-jet printed droplet on a solid substrate. Phys. Fluids 16, 3403-3414 (2004).

4. Zhai, L. et al. Patterned superhydrophobic surfaces: Toward a synthetic mimic of the Namib Desert beetle. Nano Lett. 6, 1213-1217 (2006).

5. Meuler, A. J., McKinley, G. H. \& Cohen, R. E. Exploiting topographical texture to impart icephobicity. ACS Nano 4, 7048-7052 (2010).

6. Chen, X. et al. Nanograssed micropyramidal architectures for continuous dropwise condensation. Adv. Funct. Mater. 21, 4617-4623 (2011).

7. Bird, J. C., Dhiman, R., Kwon, H-M. \& Varanasi, K. K. Reducing the contact time of a bouncing drop. Nature 503, 385-388 (2013).

8. Liu, Y. et al. Pancake bouncing on superhydrophobic surfaces. Nature Phys. 10, 515-519 (2014)

9. De Ruiter, J., Oh, J. M., van den Ende, D. \& Mugele, F. Dynamics of collapse of air films in drop impact. Phys. Rev. Lett. 108, 074505 (2012).

10. Xu, L., Zhang, W. W. \& Nagel, S. R. Drop splashing on a dry smooth surface. Phys. Rev. Lett. 94, 184505 (2005).

11. Duez, C., Ybert, C., Clanet, C. \& Bocquet, L. Making a splash with water repellency. Nature Phys. 3, 180-183 (2007).

12. Marchand, A., Chan, T. S., Snoeijer, J. H. \& Andreotti, B. Air entrainment by contact lines of a solid plate plunged into a viscous fluid. Phys. Rev. Lett. 108, 204501 (2012).

13. Mandre, S., Mani, M. \& Brenner, M. P. Precursors to splashing of liquid droplets on a solid surface. Phys. Rev. Lett. 102, 134502 (2009).

14. Mani, M., Mandre, S. \& Brenner, M. P. Events before droplet splashing on a solid surface. J. Fluid Mech. 647, 163-185 (2010).

15. Duchemin, L. \& Josserand, C. Curvature singularity and film-skating during drop impact. Phys. Fluids 23, 091701 (2011).

16. Mandre, S. \& Brenner, M. P. The mechanism of a splash on a dry solid surface. J. Fluid Mech. 690, 148-172 (2012). 
17. Driscoll, M. M. \& Nagel, S. R. Ultrafast interference imaging of air in splashing dynamics. Phys. Rev. Lett. 107, 154502 (2011).

18. Kolinski, J. M. et al. Skating on a film of air: Drops impacting on a surface. Phys. Rev. Lett. 108, 074503 (2012).

19. Van der Veen, R. C., Tran, T., Lohse, D. \& Sun, C. Direct measurements of air layer profiles under impacting droplets using high-speed color interferometry. Phys. Rev. E 85, 026315 (2012).

20. Kolinski, J. M., Mahadevan, L. \& Rubinstein, S. M. Lift-off instability during the impact of a drop on a solid surface. Phys. Rev. Lett. 112, 134501 (2014).

21. Biance, A-L., Chevy, F., Clanet, C., Lagubeau, G. \& Quéré, D. On the elasticity of an inertial liquid shock. J. Fluid Mech. 554, 47-66 (2006).

22. Couder, Y., Fort, E., Gautier, C. H. \& Boudaoud, A. From bouncing to floating: Noncoalescence of drops on a fluid bath. Phys. Rev. Lett. 94, 177801 (2005).

23. Gilet, T., Terwagne, D., Vandewalle, N. \& Dorbolo, S. Dynamics of a bouncing droplet onto a vertically vibrated interface. Phys. Rev. Lett. 100, 167802 (2008).

24. Gilet, T. \& Bush, J. W. The fluid trampoline: Droplets bouncing on a soap film. J. Fluid Mech. 625, 167-203 (2009).

25. Moláček, J. \& Bush, J. W. Drops bouncing on a vibrating bath. J. Fluid Mech. 727, 582-611 (2013).

26. Chandra, S. \& Avedisian, C. On the collision of a droplet with a solid surface. Proc. R. Soc. Lond. A 432, 13-41 (1991).

27. Tran, T., Staat, H. J. J., Prosperetti, A., Sun, C. \& Lohse, D. Drop impact on superheated surfaces. Phys. Rev. Lett. 108, 036101 (2012).

28. Richard, D. \& Quere, D. Bouncing water drops. Europhys. Lett. 50, 769-775 (2000)

29. Arayanarakool, R., Shui, L. L., van den Berg, A. \& Eijkel, J. C. T. A new method of UV-patternable hydrophobization of micro- and nanofluidic networks. Lab Chip 11, 4260-4266 (2011).
30. Tsougeni, K., Vourdas, N., Tserepi, A., Gogolides, E. \& Cardinaud, C. Mechanisms of oxygen plasma nanotexturing of organic polymer surfaces: From stable super hydrophilic to super hydrophobic surfaces. Langmuir 25, 11748-11759 (2009).

31. Mampallil, D., Burak Eral, H., Staicu, A., Mugele, F. \& van den Ende, D. Electrowetting-driven oscillating drops sandwiched between two substrates. Phys. Rev. E 88, 053015 (2013).

32. Moláček, J. \& Bush, J. W. A quasi-static model of drop impact. Phys. Fluids 24, 127103 (2012)

\section{Acknowledgements}

We thank HIPRINS for supporting this work. The project is co-sponsored by the Dutch Ministry of Economic Affairs, Agriculture and Innovation, the provinces of Overijssel, Limburg, and Noord-Brabant, and the cityregion Eindhoven (SRE).

\section{Author contributions}

J.d.R. and F.M. designed the experiments; R.L. carried out the experiments; J.d.R. and D.v.d.E. analysed the experiments; J.d.R. and F.M. wrote the manuscript.

\section{Additional information}

Supplementary information is available in the online version of the paper. Reprints and permissions information is available online at www.nature.com/reprints.

Correspondence and requests for materials should be addressed to F.M.

\section{Competing financial interests}

The authors declare no competing financial interests. 